\title{
Radial electric fields and global electrostatic microinstabilities in tokamaks and stellarators
}

\author{
L. Villard, ${ }^{1}$ A. Bottino, O. Sauter, and J. Vaclavik \\ Centre de Recherches en Physique des Plasmas, Association Euratom - Confédération \\ Suisse, Ecole Polytechnique Fédérale de Lausanne, PPB, CH-1015 Ecublens, \\ Switzerland
}

The effects of applied radial electric fields on the stability of Ion Temperature Gradient (ITG) modes and trapped particle modes are investigated with a full radius gyrokinetic formulation in both axisymmetric and helically symmetric configurations. The validity of a simplified stabilization criterion often applied to experimental analysis (the shearing rate larger than the linear growth rate) is examined for various profiles of $E \times B$ flows using an expression for the shearing rate derived for arbitrary quasisymmetric geometries [T.S. Hahm, Phys. Plasmas 4, 4074 (1997)]. The results show that the criterion holds for profiles with finite shearing rate and for toroidal, helical and slab-like ITG modes. However, it is not always applicable for trapped particle instabilities: a case is shown for which $E \times B$ flows are destabilizing. For profiles with zero shearing rate another stabilizing mechanism is found for toroidal and helical ITG modes but not for slab-like modes.

\footnotetext{
${ }^{1}$ Electronic mail: laurent.villard@epfl.ch
} 


\section{Introduction}

Microinstabilities such as the Ion Temperature Gradient mode (ITG), the Trapped Ion Mode (TIM) and the Trapped Electron Mode (TEM) are underlying the turbulent processes commonly held responsible for the anomalous transport in magnetically confined plasmas. Various theoretical and experimental results support this affirmation. It has also been shown that the presence of radial electric fields plays a pivotal role in the process $^{1-} .9$ These radial electric fields may arise from the turbulent process itself: these are the so-called "zonal flows", which appear due to modulational instability of the underlying linearly unstable mode. But the turbulence self-generated radial electric field is not necessarily the only one. An inspection of the radial force balance equation, $\mathbf{E}=-\mathbf{v}_{i} \times \mathbf{B}+\frac{1}{Z_{i} e n_{i}} \nabla P_{i}$, where $\mathbf{v}_{i}$ is the fluid ion velocity, $Z_{i} e$ is the ion charge and $P_{i}$ is the ion pressure, hints at other possibilities for a radial electric field to develop: for example by increasing the plasma toroidal rotation with tangential neutral beam injection and the application of strong heating power. In other experiments an electric field in the plasma has been directly created ${ }^{6,7}$ with the application of an electrode bias. The general observation is that there is a correlation between improved confinement, reduction of turbulence levels and rise of radial electric fields.

The picture that emerges is that when equilibrium gradients exceed a certain critical value microinstabilities grow. When their amplitude gets large enough nonlinear coupling occurs to the $m=0, n=0$ component (the zonal flows). The effect of these radial electric fields is to saturate the turbulence to a finite level which is then responsible for the development of an anomalous heat flux. The mechanism is understood as a shearing of the unstable modes by the $E \times B$ flows. When the shearing rate of the $E \times B$ flow exceeds a certain value the turbulence is supressed, thus giving rise to various types of improved confinement regimes (H-mode, internal transport barriers, etc). It is nowadays clear that any realistic future fusion device will have to rely on at least one of these improved confinement regimes.

Previous works ${ }^{1,8,9}$ have expressed a stabilization criterion by $E \times B$ flows based on the decorrelation time of turbulence. In Ref. ${ }^{8}$ the shearing rate is given as :

$$
\omega_{E \times B} \approx \rho \frac{\partial}{\partial \rho}\left(\frac{v_{E}}{\rho}\right),
$$

where $v_{E}$ is the $E \times B$ drift velocity, $\rho$ is the plasma minor radius. The interpretation is rather simple: the shearing rate is equal to the shear in the poloidal angular $E \times$ $B$ velocity, and when this shear exceeds the inverse turbulence decorrelation time the mode is stabilized. However, this expression is only valid for circular, large aspect ratio tokamak configurations and omits the magnetic shear effect. In Ref. ${ }^{1}$ a more general expression, valid for any axisymmetric geometry, is proposed. It is further generalized 
to quasisymmetric configurations. ${ }^{9}$ Neglecting parallel flows, which are not considered in the present paper, it can be written for both axisymmetric and helically symmetric systems as

$$
\omega_{E \times B}=\frac{\Delta \psi}{\Delta \varphi} \frac{d^{2}}{d \psi^{2}} \Phi_{0}(\psi) \approx \frac{s(d \psi / d s)}{q} \frac{d^{2}}{d \psi^{2}} \Phi_{0}(\psi),
$$

where, for the axisymmetric case, $\psi$ is the poloidal flux, $s=\sqrt{\psi}, q$ is the safety factor, $\mathbf{E}=-\nabla \Phi_{0}(\psi)$ is the zonal component of the electric field, $\Delta \psi /|\nabla \psi|$ and $R \Delta \varphi$ are the radial and toroidal correlation lengths of turbulence, respectively. For the helically symmetric case $\psi$ is the helical flux and $q=1 /(\iota-1)$, where $\iota$ is the rotational transform per period. [Note that the second equality in (2) is obtained via two assumptions: first, that the turbulent eddies tend to isotropize perpendicularly to the magnetic field and second, that the turbulence tends to align with magnetic field lines]. To get an insight into the meaning of Eq.(2) it is useful to write it in the circular large aspect ratio approximation:

$$
\omega_{E \times B} \approx \frac{\rho}{q} \frac{\partial}{\partial \rho}\left(q \frac{v_{E}}{\rho}\right) .
$$

This means that the shearing rate is not simply the shear in the poloidal angular $E \times B$ velocity, but it also includes a combined effect of the magnetic shear with the value of this velocity.

For practical purposes a simplified criterion is often used in the literature, in particular for the analysis of experiments: $\left|\omega_{E \times B}\right|>\gamma_{0}$, where $\omega_{E \times B}$ is the shearing rate of the $E \times B$ flow and $\gamma_{0}$ is the maximum linear growth rate in the absence of flows, while the proper comparison of the shearing rate should be with the inverse decorrelation rate of turbulence. Also, it is appropriate to recall the basic difference between zonal flows, which are self-generated by the turbulence and are fluctuating quantities, and applied $E \times B$ flows which are considered as equilibrium quantities. This paper focuses on the latter and examines the validity of the simplified shearing rate criterion for its effect on various types of microinstabilities.

The remainder of the article is organized as follows. In Section 2 the global gyrokinetic model is introduced for both axisymmetric and helically symmetric configurations, using a linearized $\delta f, \delta \phi$ approach, and a finite element, Particle-In-Cell (PIC) discretization scheme. In Section 3 the model is applied to toroidal-ITG modes with different profiles of applied $E \times B$ flows. In section 4 the trapped ion and trapped electron modes are studied. In section 5 we show the first application to a particular helical configuration. Finally, the main conclusions of the paper are presented in Section 6 . 


\section{Global gyrokinetic model in axisymmetric and he- lical geometries}

We consider an equilibrium magnetic configuration with the representation

$$
\mathbf{B}=F \mathbf{u}+\nabla \psi \times \mathbf{u}
$$

For an axisymmetric case, $\mathbf{u}=\nabla \varphi, \varphi$ is the toroidal angle, $\psi$ is the poloidal flux and $F=F(\psi)$ is the toroidal current flux function. For a helical configuration, $\mathbf{u}=\left(h r \mathbf{e}_{\varphi}+\right.$ $\left.\mathbf{e}_{z}\right) /\left(1+h^{2} r^{2}\right),(r, \varphi, z)$ are the usual cylindrical coordinates, $h$ is the helicity and $\psi$ is the helical flux per unit length in the $z$ direction; all scalar equilibrium quantities are functions of two variables: $r$ and $\zeta=\varphi-h z$. We define $\left(x^{\prime}, y^{\prime}\right)$ as $\left(x^{\prime}=r, y^{\prime}=z\right)$ for the axisymmetric case, and as $\left(x^{\prime}=r \cos \zeta, y^{\prime}=r \sin \zeta\right)$ for the helical case. A radial magnetic coordinate is defined as $s=\left(\left(\psi-\psi_{0}\right) /\left(\psi_{a}-\psi_{0}\right)\right)^{1 / 2}$ and a poloidal angle $\theta=\arctan \left(y^{\prime} /\left(x^{\prime}-x_{0}^{\prime}\right)\right)$, where the subscripts 0 and $a$ indicate the magnetic axis and the plasma boundary, respectively. The safety factor $q$ and the straight field line coordinate $\chi$ are defined as

$$
q=\frac{1}{L} \int_{0}^{2 \pi} \frac{\mathbf{B} \cdot \nabla \xi}{\mathbf{B} \cdot \nabla \theta} d \theta, \quad \chi=\frac{2 \pi}{q L} \int_{0}^{\theta} \frac{\mathbf{B} \cdot \nabla \xi}{\mathbf{B} \cdot \nabla \theta^{\prime}} d \theta^{\prime}
$$

where $\xi$ is the ignorable coordinate and $L$ is the period length $(\xi=\varphi, L=2 \pi$ for the axisymmetric case; $\xi=z, L=2 \pi / h$ for the helical case). Note that the rotational transform per period length is $\iota=1 / q$ for axisymmetric configurations and $\iota=1+1 / q$ for helical configurations. The poloidal (respectively helical) flux $\psi$ is a solution of the Grad-Shafranov equation which for helical systems is

$$
\frac{1}{r} \frac{\partial}{\partial r}\left(r|u|^{2} \frac{\partial \psi}{\partial r}\right)+\frac{1}{r^{2}} \frac{\partial^{2} \psi}{\partial \zeta^{2}}=-\left(\frac{d P}{d \psi}+|u|^{2} F \frac{d F}{d \psi}+2 h|u|^{4} F\right) .
$$

[Note that the axisymmetric Grad-Shafranov equation is obtained by formally setting $h=0$ in the above equation and replacing $\zeta$ with $z$.] In this paper we shall consider low $\beta$ equilibria. In the helical case we further assume current-free configurations for which we have the analytical solution

$$
\psi=\frac{1}{2} b_{0} h r^{2}-c_{0} \ln r-r \sum_{l} b_{l} I_{l}^{\prime}(l h r) \cos (l \zeta), \quad F=b_{0}+h c_{0} .
$$

We assume equilibrium profiles of density $n_{0}(\psi)$, temperatures $T_{i}(\psi)$ and $T_{e}(\psi)$ and potential $\Phi_{0}(\psi)$ and study perturbations of the ion and electron distribution functions $\delta f=f-f_{0}$ and electrostatic potential $\delta \phi=\phi-\Phi_{0}$. We consider the gyrokinetic model with the usual ordering: $\omega / \Omega \sim k_{\|} / k_{\perp} \sim e \delta \phi / T_{e} \sim \rho_{L} / L_{n} \sim \rho_{L} / L_{T} \sim \mathcal{O}\left(\epsilon_{g}\right)$, where $\rho_{L}$ is the ion Larmor radius, $\Omega$ is the ion cyclotron frequency, $L_{E}^{-1}=|\nabla \ln E|, E=n_{0}, T$. 
Another small parameter is $\rho_{L} / L_{B} \sim \mathcal{O}\left(\epsilon_{B}\right)$, with $L_{B}=B /|\nabla B|$. Consistent with the gyrokinetic ordering, the perturbations of interest have $k_{\|}<<k_{\perp}$. We use this property to extract the poloidal phase variation of the mode by writing the perturbed quantities as

$$
\delta f(\mathbf{x}, t)=\tilde{\delta f}(s, \theta, t) e^{i S}, \quad \delta \phi(\mathbf{x}, t)=\tilde{\delta \phi}(s, \theta, t) e^{i S}
$$

with

$$
S(s, \theta, z, t)=m_{0} \chi\left(s_{0}, \theta\right)+n \varphi-\omega_{0} t
$$

for the axisymmetric case and

$$
S(s, \theta, z, t)=m_{0} \chi\left(s_{0}, \theta\right)+k z-\omega_{0} t
$$

for the helical case. In Eqs. (9)(10) $s_{0}$ is a reference magnetic surface usually taken near the maximum mode amplitude position and $m_{0}$ is an integer chosen as close as possible to $n q\left(s_{0}\right)$. This technique of extracting the rapidly varying poloidal phase implies that the transformed quantities vary much more smoothly and thus much less numerical resolution is required for a given accuracy with virtually no upper bound for the poloidal mode number: a gain of up to two orders of magnitude in numerical performance can be attained. ${ }^{10}$

From Ref. ${ }^{11}$ we obtain the following linearized system of equations:

$$
\begin{gathered}
\frac{d \mathbf{R}}{d t}=v_{\|} \mathbf{e}_{\|}+\mathbf{v}_{E}\left(1-\frac{1}{\Omega} \mathbf{e}_{\|} \cdot \nabla \times\left(\mathbf{v}_{E}+v_{\|} \mathbf{e}_{\|}\right)\right) \\
+\frac{1}{\Omega} \mathbf{e}_{\|} \times\left(v_{\|} \nabla \times\left(\mathbf{v}_{E}+v_{\|} \mathbf{e}_{\|}\right) \times \mathbf{e}_{\|}+\mu \nabla B+\frac{1}{2} \nabla v_{E}^{2}\right), \\
\frac{d v_{\|}}{d t}=-\mathbf{e}_{\|} \cdot\left(\mu \nabla B+\frac{1}{2} \nabla v_{E}^{2}\right)-\mathbf{v}_{E} \cdot\left(\mathbf{e}_{\|} \times \nabla \times\left(\mathbf{v}_{E}+v_{\|} \mathbf{e}_{\|}\right)\right), \\
\frac{d v_{\perp}}{d t}=\frac{\mu}{v_{\perp}}\left(\mathbf{v}_{E}+v_{\|} \mathbf{e}_{\|}\right) \cdot \nabla B, \\
\frac{d \tilde{\delta f}}{d t}+i \frac{d S}{d t} \tilde{\delta f}=\frac{-\nabla<\tilde{\delta \phi}>}{B} \\
\cdot\left[\left(\nabla f_{0}+\frac{\mu}{v_{\perp}} \frac{\partial f_{0}}{\partial v_{\perp}} \nabla B-\frac{\partial f_{0}}{\partial v_{\|}} \mathbf{e}_{\|} \times \nabla \times\left(\mathbf{v}_{E}+v_{\|} \mathbf{e}_{\|}\right)\right) \times \mathbf{e}_{\|}-\Omega \frac{\partial f_{0}}{\partial v_{\|}} \mathbf{e}_{\|}\right]
\end{gathered}
$$

where $\mathbf{v}_{E}=\mathbf{e}_{\|} \times \nabla \Phi_{0} / B, \Omega$ is the cyclotron frequency, $\mu=v_{\perp}^{2} / 2 B$. The gyro-averaged perturbed electric field at the gyro-center position $\mathbf{R}=\mathbf{x}+\boldsymbol{\rho}_{L}$ is

$$
\nabla<\tilde{\delta \phi}>=-\frac{1}{2 \pi} \int(\mathcal{G} \tilde{\delta \phi}) \delta\left(\mathbf{x}-\mathbf{R}+\boldsymbol{\rho}_{L}\right) e^{i S(\mathbf{x})-i S(\mathbf{R})} d \mathbf{x} d \alpha
$$

where $\mathcal{G}=i \nabla S+\nabla$ and $\alpha$ is the gyroangle. 
While ions are fully gyrokinetic we choose to model trapped electrons using the driftkinetic approximation obtained setting $\rho_{L e}=0$ in the above equations. Passing electrons are assumed to respond adiabatically to the perturbation. The system of equations is closed invoking the quasi-neutrality condition, $\delta n_{e}=\delta n_{i}$. The ion polarization density is written using a Padé approximation which has been shown to be valid for $k_{\perp} \rho_{L}<2 .{ }^{12}$ The quasi-neutrality equation then becomes

$$
\left(1-\mathcal{G}_{\perp} \frac{T_{i}}{m_{i} \Omega^{2}} \mathcal{G}_{\perp}\right)\left(\tilde{\delta}_{i}-\tilde{n}_{e}\right)=\mathcal{G}_{\perp}\left(\frac{n_{0}}{B \Omega} \mathcal{G}_{\perp} \tilde{\delta \phi}\right)
$$

The above set of equations is discretized using "particles" (gyrocentre tracers) ${ }^{13,14}$ and finite elements on a magnetic coordinate system $(s, \theta) \cdot{ }^{10}$ As we shall consider cases with $v_{E}<<v_{t i}$ the terms of order $v_{E}^{2} / v_{t i}^{2}$ and $v_{E} v_{d} / v_{t i}^{2}$ have been neglected, where $v_{d}$ is the magnetic drift velocity and $v_{t i}$ is the ion thermal velocity.

Besides the usual numerical convergence tests with number of tracers and mesh size the conservation properties of the equations can be checked. For particles we have three constants of motion: the energy $\varepsilon=m v_{\|}^{2} / 2+m \mu B+Z_{i} e \Phi_{0}$, the magnetic moment $\mu=v_{\perp}^{2} / 2 B$ and the conjugate momentum $\Psi_{0}$. A unified expression for $\Psi_{0}$ in axisymmetric or helically symmetric configurations can be obtained: [noting that in axisymmetric geometry the corresponding one-form for particle motion is invariant in the toroidal direction $\varphi$, whereas in helical geometry it is invariant in the helical direction $\mathbf{u}$.] Using the magnetic representation (4) we obtain for both geometries

$$
\Psi_{0}=\psi+\frac{m_{i}}{Z_{i} e} v_{\|} \frac{F}{B}=\text { const } .
$$

The power balance can also be verified: the time rate of change of the field energy equals the power transfer from the particles: $d E_{\text {field }} / d t=-d E_{k i n} / d t$ or

$$
\frac{d}{d t} \frac{q_{i}}{2} \int\left(\frac{n_{0} e}{T_{e}}<\delta \phi>^{2}+\frac{n_{0}}{B \Omega}\left|<\nabla_{\perp} \delta \phi>\right|^{2}\right) d \mathbf{x}=q_{i} \int \delta f \frac{d \mathbf{R}}{d t} \cdot<\nabla \delta \phi>d \mathbf{R} d \mathbf{v} .
$$

\section{Stabilization of toroidal ITG mode with various profiles of $E \times B$}

We consider a circular cross-section tokamak with the following parameters: $R_{0}=1.19$ $(\mathrm{m}), a=0.21(\mathrm{~m}), B_{0}=1(\mathrm{~T}), q(s)=1.25+3 s^{2}$, constant density, $T_{i}=T_{e}$, and a temperature profile given by

$$
\frac{d \ln T}{d s}=-\frac{1}{L_{T}} \cosh ^{-2}\left(\frac{s-s_{0}}{\Delta_{T}}\right)
$$

with the position of maximum gradient at $s_{0}=0.617, L_{T}=0.77, \Delta_{T}=0.31$. Ions are hydrogen. The parameters have been chosen so that the maximum growth rate in the 
absence of $E \times B$ flow is for a toroidal wave number $n=7$ and the corresponding mode is a toroidal ITG.

In order to discriminate between expressions of the shearing rate given in Eq.(1) and in Eq.(2), respectively, the following profiles of $E \times B$ are chosen. First,

$$
\mathbf{v}_{E}=M v_{t h i 0} \frac{\rho}{\rho_{0}} \mathbf{e}_{\theta}
$$

where $M$ is the specified Mach number, $v_{t h i 0}$ is the ion thermal velocity at $s=s_{0}, \rho$ is the minor radius, $\rho_{0}$ is the minor radius of the magnetic surface $s=s_{0}$, . This specifies a shearless profile of $E \times B$ flow and therefore a zero shearing rate according to Eq.(1) but a nonzero shearing rate according to Eq.(2). Second,

$$
\mathbf{v}_{E}=M v_{t h i 0} \frac{B_{c}}{B} \frac{|\nabla \psi| a}{2 \psi_{s} s_{0}} \mathbf{e}_{b}
$$

where $B_{c}$ is the magnetic field on axis, $a$ is the average minor radius and $\mathbf{e}_{b}=\mathbf{e}_{\|} \times \mathbf{e}_{n}$. This profile is constructed from $\mathbf{v}_{E}=\mathbf{e}_{\|} \times \nabla \psi\left(d \Phi_{0} / d \psi\right) / B$ with a constant profile of $d \Phi_{0} / d \psi$, so that the shearing rate of this $E \times B$ flow is zero according to Eq.(2) but not according to Eq.(1). A third profile is defined by

$$
\mathbf{v}_{E}=M v_{t h i 0} \frac{B_{c}}{B} \frac{|\nabla \psi| a}{2 \psi_{s} s_{0}}\left(s-s_{0}\right) \mathbf{e}_{b}
$$

so that the shearing rate is nonzero according to both Eq.(1) and Eq.(2). Note that profiles of Eqs.(20) and (21) have the same finite value $v_{E}=M v_{t h i 0}$ at $s=s_{0}$, whereas that of Eq.(22) is zero.

Simulation results in which all electrons have been assumed to respond adiabatically are shown in Figure 1 in which the highest growth rate is plotted against the Mach number. The shearless profile of $E \times B$ flow, Eq.(20), shown with filled symbols, leads to a strong stabilization of the toroidal ITG mode. The Mach number required for stabilization would be infinity if Eq.(1) is used, whereas it is $|M|=0.016$ if Eq.(2) is used. The latter is clearly in a much better agreement with the simulation results. The physical picture of the stabilization in this case is that, although the $E \times B$ flow is shearless, its shearing rate is nonzero. The effect can be observed on the structure of the perturbed potential of the mode: the radially extended structures, typical for toroidal ITGs, are getting tilted poloidally, ${ }^{15}$ in a similar manner as was found previously for toroidal drift-waves ${ }^{4}$ although with a different $E \times B$ flow profile. Note that the tilting effect is studied in detailed in Ref. ${ }^{5}$ in the vicinity of a shear reversal region.

Results for the zero shearing rate profile of $E \times B$ flow, Eq.(21), are shown with open symbols in Figure 1. The Mach number required for stabilization would be $|M|=0.016$ if Eq.(1) is used, and infinity if Eq.(2) is used. Thus none of the expressions for the 
shearing rate appears to be applicable. However, an inspection of the mode shown in Figure 2 (left, with $M=0$; right, with $M=-0.034$ ) shows that indeed the radially extended structures are not tilted by the flow: note that the poloidal position $\theta_{0}$ for which the mode structure is radially aligned is not changed by this radial electric field profile. Another mechanism plays a stabilizing role here: the position of maximum mode amplitude is shifted away from the unfavourable $\nabla B$ region, from $\theta \approx 0$ for $M=0$ to $\theta \approx-\pi / 2$ for $M=-0.034$.

The dashed line in Figure 1 are results for the sheared flow profile with zero value at $s=s_{0}$, Eq.(22). In this case the tilting effect on the mode structure is effective in stabilizing the mode, in agreement with previous studies made for similar profiles. ${ }^{4,5}$ The application of the simplified stabilization criterion would give in this case $|M|=0.25$.

We conclude from the above studies that Eq.(2) should be used for the expression of the $E \times B$ shearing rate and that the simplified stability criterion $\omega_{E \times B} \approx \gamma_{0}$ holds rather well for this toroidal-ITG case. In particular the combined effect of the magnetic shear with the value of $v_{E}$ is clearly illustrated: for the case shown in Figure 1 the shearless $v_{E}$ profile is the most effective in stabilizing the toroidal ITG. We have also found that another stabilizing mechanism for toroidal ITG modes exists even for zero shearing rate of the $E \times B$ flow. This acts by shifting the position of maximum mode amplitude towards more favourable $\nabla B$ regions.

Using the same parameters but considering drift-kinetic trapped electrons and adiabatic passing electrons yields the same qualitative results for the $E \times B$ stabilization of the toroidal ITG mode. The only marked difference is the overall higher values of growth rates as compared to the case of full adiabatic electrons.

\section{Stabilization and destabilization of TIM and TEM}

Turning now to trapped particle instabilities we consider the following tokamak case: $R_{0}=1.5 \mathrm{~m}, a=0.5 \mathrm{~m}, B_{0}=1 \mathrm{~T}$, hydrogen ions, $T_{i}=T_{e}, T_{0}=5 \mathrm{keV}, L_{T} / a=0.3$, $L_{n} / a=3, s_{0}=0.705 \Rightarrow \rho_{0} / a=0.6$. The $q$ profile $q(\rho / a)=1+2.315(\rho / a)^{3}$ is such that $q\left(s_{0}\right)=1.5$ and the magnetic shear at $s=s_{0}$ is unity. For these parameters the most unstable mode is a Trapped Ion Mode (TIM) with $n=8 .{ }^{16}$ Adding a shearless $E \times B$ flow, Eq.(20), and considering purely adiabatic electrons, we obtain the growth rates of the most unstable modes of Figure 3. The remarkable result is that although the most unstable mode at $\mathrm{Mach}=0$ is stabilized with small values of Mach, other modes are destabilized even to higher growth rates than without $E \times B$ flow. The time evolution approach adopted in this paper allows us to find the most unstable mode only. With a spectral approach it was shown in Ref. ${ }^{16}$ that indeed there are several unstable modes at 
$\mathrm{Mach}=0$. Using the same global spectral code we have computed the unstable spectra in the presence of $E \times B$ flows. The results show that the TIM, initially most unstable at $\mathrm{Mach}=0$, is stabilized with increasing $v_{E}$. But before it gets completely stable another mode is destabilized and becomes the most unstable mode at a finite Mach value. This other mode is then also stabilized but a third mode then becomes unstable. The overall effect is that complete stabilization is not reached, unlike the toroidal-ITG case shown in the previous section.

Considering now drift-kinetic trapped electrons and passing adiabatic electrons we obtain the growth rates and frequencies of Figure 4. As noted in the previous case the inclusion of the trapped electron response brings an overall destabilization as compared to the purely adiabatic electron response case. The remarkable feature is the strong destabilization of a Trapped Electron Mode (TEM) for $M<-0.01$ : the change in the frequency sign is clearly visible. Note that negative Mach numbers correspond to negative radial electric fields. We have verified that the TEM destabilization is not due to a direct effect of the $E \times B$ flow on the electron trajectories: virtually the same results are obtained when including or neglecting the $v_{E}$ term in the equation of motion of electrons. So the destabilization mechanism is more subtle. With trapped electrons and positive $E_{r}$ the same qualitative results are obtained as with adiabatic electrons, i.e. other TIMs are destabilized; the inclusion of trapped electron dynamics is in this case quantitative: all growth rates are higher as compared to the full adiabatic electron case. With trapped electrons and negative $E_{r}$ one TIM is stabilized but a TEM is destabilized to even higher growth rates than the case without flow.

The general conclusion of these investigations is that there are cases for which radial electric fields can be overall destabilizing rather than stabilizing, in particular when trapped particle dynamics is taken into account.

\section{$5 \quad$ Stabilization of ITG modes in a helical stellarator}

We consider a helically symmetric configuration given by Eq.(7) with the parameters $h=2.905\left(m^{-1}\right), b_{0}=1(T), c_{0}=0.11(T m), b_{1}=0.5(T m), b_{2}=-0.06(T m)$. This results in a bean shaped plasma cross-section with a helical magnetic axis of radius $x_{0}^{\prime}=0.26(\mathrm{~m})$, an average minor radius $\langle a\rangle=0.21(\mathrm{~m})$ and an elongation of 2.02 . The configuration is shown in Figures 5 and 6 . The most characteristic feature of this equilibrium is the virtual absence of magnetic shear, with $q=-1.507$, hence a rotational transform per $2 \pi / h$ longitudinal length of $\iota=0.3365$, constant up to $10^{-3}$ across the plasma. The other property of this heliac configuration is that it does have magnetic field gradients comparable in magnitude to those in a tokamak of aspect ratio 5 - albeit 
with a different symmetry of course. The temperature and density profiles are the same as for the tokamak results for the toroidal-ITG studies presented in the previous section.

Before we analyze the effects of $E \times B$ flows we first show in Figure 7 the growth rates and frequencies of the most unstable mode as a function of the longitudinal wavenumber $k$. The spectrum exhibits an alternance of stable windows near resonant wavenumbers $\left(k_{\|}=0\right.$, vertical dotted lines), slab-like ITG modes (noted 'S') and helical-ITG modes (noted 'H'), in a pattern which repeats itself. Note the jumps in frequency at the S-H transitions, indicating that the ' $\mathrm{S}$ ' and ' $\mathrm{H}$ ' ITGs are different modes. For ' $\mathrm{S}$ ' modes, the frequency is roughly proportional to $\left|k_{\| m}\right|=|h m / q+k|$. The poloidal Fourier decomposition of the perturbed potential shows a single dominant mode number $m$. The ' $S$ ' modes are characterized by a destabilizing parallel dynamics and this is reflected in the parallel contribution to the wave-particle power exchange $\left(\int j_{\|} E_{\|} d \mathbf{x}>0\right)$. As $\left|k_{\|}\right|$ increases, ion Landau damping increases and this is the responsible mechanism for the levelling off of the growth rate of 'S' modes. For 'H' modes, however, the poloidal Fourier decomposition of the perturbed potential shows a mixture of neighbouring $m$ values with approximately equal amplitude: the mode tends then to have its largest amplitude in the most unfavourable $\nabla B$ drift region, and the parallel wavenumber is approximately equal to $h /\left(q \sqrt{1+h^{2} x_{0}^{\prime 2}}\right)$ independently of $k$. The 'H' modes are characterized by a stabilizing parallel dynamics $\left(\int j_{\|} E_{\|} d \mathbf{x}<0\right)$ and a destabilizing perpendicular dynamics $\left(\int \mathbf{j}_{\perp} \cdot \mathbf{E}_{\perp} d \mathbf{x}>0\right)$. We remark a decrease of the frequency of the 'H' modes with $k$ : this is due to a global effect: the amplitude of the perturbed potential moves radially outward where the temperature is lower. As for the stable windows, it has been shown in ${ }^{17}$ that they are due to an effect of the $\nabla B$ drifts which, in heliac configurations, are stabilizing modes with small $k_{\|}$. This unique property might be responsible for the resonant behaviour of transport with rational values of the rotational transform in such shearless systems.

Adding $E \times B$ flows to the system we show in Figure 8 their effect on the growth rate of a slab-like mode and in Figure 9 on a helical-ITG mode. Two different profiles of flow are considered: a zero shearing rate profile with $d \Phi_{0} / d \psi=$ const (open symbols) and a linear profile of $d \Phi_{0} / d \psi$ with zero value at $s=s_{0}$ (dashed line). For 'S' modes in Figure 8 the shearing rate criterion, using Eq.(2) for the linear profile would give stabilization at $|M| \approx 0.027$. For ' $\mathrm{H}$ ' modes in Figure 9 it would give $|M| \approx 0.03$. We observe that for both cases the global simulation results agree well with the simplified stabilization criterion. An investigation of the perturbed potential (Figures 10-12) shows that the effect of the profile of flow with nonzero shearing rate is to twist the radially extended structures. For $M=0.01$ these structures are aligned at $\theta=0$ (Figure 11) and the growth rate is maximal. For higher Mach numbers (Figure 12) the structures are 
misaligned and the growth rate decreases. Thus the mechanism is similar to what was found for the toroidal ITG case. ${ }^{4}$

For the zero shearing rate profile the criterion would give no stabilization. Indeed, for slab-like modes our results show virtually no effect (Figure 8, open symbols). On the other hand, there is a stabilization of the helical-ITG (Figure 9, open symbols). The mechanism works similarly to the toroidal-ITG case in tokamaks, namely the finite value of $v_{E}$ pushes the position of maximum mode amplitude in the poloidal direction towards more favourable $\nabla B$ drift regions.

The main results of this section can be summarized as follows. For $v_{E}$ profiles with finite shearing rate, the simplified stabilization criterion $\left|\omega_{E \times B}\right| \approx \gamma_{0}$ appears to be applicable for the heliac geometry considered here, when the appropriate expression for the shearing rate $^{9}$ is used. Zero shearing rate $v_{E}$ profiles can stabilize helical-ITG modes in a similar way as the toroidal-ITG mode in tokamaks. However, they have virtually no stabilizing effect on slab-like modes; this might prove of importance since in helical systems the presence of slab-like modes is much more prominent than in axisymmetric toroidal systems.

\section{Conclusions}

The crucial role of applied radial electric fields on microinstabilities has been evidenced in tokamak and stellarator configurations within the frame of the gyrokinetic model and using full radius linearized $\delta f, \delta \phi$ simulations.

- Three classes of ITG modes (toroidal, helical and slab-like) and two classes of trapped particle modes (TIM and TEM) have been investigated, on which $E \times B$ flows have different effects.

- $E \times B$ flows with zero shearing rate stabilize toroidal and helical ITG modes by shifting the position of maximum mode amplitude away from the unfavourable grad-B drift region. They do not tilt the radially elongated structures of these modes.

- $E \times B$ flows with nonzero shearing rate stabilize toroidal, helical and slab-like ITG modes. In these cases the simplified criterion $\left|\omega_{E \times B}\right| \approx \gamma_{0}$ is verified, except for a slight asymmetry in the sign of radial electric field. The effect of tilting the radial structures is similar to what was found previously for the toroidal $\eta_{i}$ mode. ${ }^{4}$

- The expression for the shearing rate that should be used in the simplified stabilization criterion in both axisymmetric and helical configurations is ${ }^{9} \omega_{E \times B}=$ 
$(s / q)(d \psi / d s)\left(d^{2} \Psi_{0} / d \psi^{2}\right)$, where $\psi$ is the poloidal flux, respectively helical flux, and $s=\sqrt{\psi}$. In the circular, large aspect ratio axisymmetric case it is $\omega_{E \times B} \approx$ $(\rho / q)(\partial / \partial \rho)\left(q v_{E} / \rho\right)$, where $\rho$ is the minor radius. The shearing rate is not simply the shear of the $E \times B$ flow: magnetic shear combined with a finite value of $v_{E}$ contributes to the shearing rate. For example, a shearless $v_{E}$ profile in a configuration with finite magnetic shear is found to be very effective in stabilizing the toroidal ITG mode.

- The effect of $E \times B$ flows on TIMs is more complex: as one TIM is stabilized, other modes show up with increasing flow and the overall effect can even be destabilizing.

- When trapped electron dynamics is taken into account the $E \times B$ flows can destabilize TEMs. An asymmetry in the sign of radial electric field was found. TEMs are destabilized for negative radial electric fields, whereas TIMs are destabilized for positive radial electric fields.

- For the first time, the effect of $E \times B$ flows on ITG modes in a helically symmetric configuration has been shown with a global gyrokinetic simulation. For $v_{E}$ profiles with finite shearing rate the simplified stabilization criterion $\left|\omega_{E \times B}\right| \approx \gamma_{0}$ appears to hold.

Finally, although the present work suggests some similarities between the axisymmetric tokamak and the helical stellarator as far as the effects of $E \times B$ flows are concerned, it is still premature to conclude that anomalous transport in stellarators is predicted to be similar to that in tokamaks. One difference is that optimized advanced stellarators, such as $\mathrm{W}-7 \mathrm{X}^{18}$ or $\mathrm{HSX}^{19}$ have much less toroidal curvature and also much less particle trapping. Therefore there is a basic difference: in tokamaks the most unstable modes are interchange-like toroidal-ITG modes, or TIMs or TEMs, whereas in W-7X or HSX stellarators the most unstable modes have more a slab-like character. ${ }^{20,21}$ For the particular shearless helical system analyzed in this paper we have found a remarkable resonant behaviour of microinstabilities with rational values of the rotational transform. For stellarator configurations with shear this is not the case. ${ }^{20,21}$ We have also found that slab-like ITG modes, which are much more prominent in helical systems than in axisymmetric systems, do not react in the same way to the presence of $E \times B$ flows having zero shearing rate. Full radius nonlinear gyrokinetic simulations in the appropriate geometries may well be required to determine whether this has consequences on the level of turbulence-driven anomalous heat flux.

\section{Acknowledgment}

This work was partly supported by the Swiss National Science Foundation. 


\section{References}

[1] T.S. Hahm and K.H. Burrell, Phys. Plasmas 2, 1648 (1995).

[2] P.H. Diamond, Y.-M. Liang, B.A. Carreras, and P.W. Terry, Phys. Rev. Lett. 72, 2565 (1994).

[3] K.H. Burrell, Phys. Plasmas 6, 4418 (1999).

[4] J.Y. Kim, Y. Kishimoto, M. Wakatani, and T. Tajima, Phys. Plasmas 3, 3689 (1996).

[5] Y. Kishimoto, J.Y. Kim, W. Horton, et al., Nucl. Fusion 40, 667 (2000).

[6] R.J. Taylor, M.L. Brown, B.D. Fried, H. Grote, J.R. Liberati, G.J. Morales, P. Pribyl, D. Darrow, and M. Ono, Phys. Rev. Lett. 63, 2365 (1989).

[7] R.R. Weynants, S. Jachmich, and G. Van Oost, Plasma Phys. Controlled Fusion 40, 635 (1998).

[8] H. Biglari, P.H. Diamond, and P.W. Terry, Phys. Fluids B 2, 1 (1990).

[9] T.S. Hahm, Phys. Plasmas 4, 4074 (1997).

[10] M. Fivaz, S. Brunner, G. de Ridder, O. Sauter, T.M. Tran, J. Vaclavik, L. Villard, and K. Appert, Comput. Phys. Commun. 111, 27 (1998).

[11] T.S. Hahm, Phys. Plasmas 3, 4658 (1996).

[12] A. Bottino, T.M. Tran, O. Sauter, J. Vaclavik, and L. Villard, in Theory of Fusion Plasmas, International Workshop, Varenna, August-September 2000 (Editrice Compositori, Società Italiana di Fisica, Bologna, 2000), p. 327.

[13] A.M. Dimits and W.W. Lee, J. Comput. Phys. 107, 309 (1993).

[14] S.E. Parker and W.W. Lee, Phys. Fluids B 5, 77 (1993).

[15] M. Maccio, J. Vaclavik, and L. Villard, Phys. Plasmas 8, 895 (2001).

[16] G.L. Falchetto, J. Vaclavik, M. Maccio, S. Brunner, and L. Villard, Phys. Plasmas 7, 1196 (2000).

[17] L. Villard, J. Vaclavik, G. Jost, M. Maccio and W.A. Cooper, in Theory of Fusion Plasmas, International Workshop, Varenna, August-September 1998 (Editrice Compositori, Società Italiana di Fisica, Bologna, 1999), p. 427. 
[18] W. Lotz, J. Nührenberg, and C.Schwab, Proceedings of the 13th International Conference on Plasma Physics and Controlled Nuclear Fusion Research, Washington, 1990 (IAEA, Vienna, 1991).

[19] F.S.B. Anderson, A.F. Almagri, D.T. Anderson, P.G. Matthews, J.N. Talmadge, and J. Leon Shohet, Trans. Fusion Technol. 27, 273 (1995).

[20] G. Jost, T.M. Tran, W.A. Cooper, L.Villard, and K. Appert, Phys. Plasmas 8, 3321 (2001).

[21] S. Allfrey, A. Bottino, R. Hatzky, G. Jost, and L. Villard, Proceedings of Contributed Papers, 28th European Physical Society Conference on Controlled Fusion and Plasma Physics, Madeira, Portugal, 2001, edited by C. Silva, C. Varandas, and D. Campbell (European Physical Society, Geneva, 2001), P5.042. 


\section{List of Figures}

1. Growth rates of the most unstable toroidal ITG mode in a tokamak versus Mach number of the $E \times B$ flow, for a shearless flow profile, Eq.(20), (filled symbols) and for a zero shearing rate flow profile, Eq.(21), (open symbols). The dashed line corresponds to a sheared flow profile with zero value at $s_{0}$.

2. Perturbed potential for a zero shearing rate profile of $E \times B$ flow with Mach=0 (left) and $\mathrm{Mach}=-0.034$ (right). The position of maximum mode amplitude is shifted away from the unfavourable grad-B region but there is no tilt of the radially elongated structures.

3. Growth rates of the most unstable Trapped Ion Mode in a tokamak versus Mach number of the $E \times B$ flow, for a shearless flow profile, Eq.(20), and assuming adiabatic electrons.

4. Growth rates (top) and frequencies (bottom) of the most unstable mode in a tokamak versus Mach number of the $E \times B$ flow, for a shearless flow profile, Eq.(20), and assuming drift-kinetic trapped electrons. Positive frequency means a phase velocity in the electron diamagnetic direction and therefore a Trapped Electron Mode.

5. Outer magnetic surface of the heliac configuration.

6. Cross-section of the heliac showing magnetic surfaces (continuous lines) and mod-B contours (dashed lines).

7. Growth rate (top) and frequency (bottom) of the most unstable mode in a heliac configuration vs longitudinal mode number. The vertical dotted lines correspond to resonant wavenumbers $\left(k_{\|}=0\right)$. Labels 'S' and 'H' mark slab-like and helical-ITG modes, respectively.

8. Growth rates of slab-like ITG modes vs Mach number in a $2 \mathrm{D}$ heliac for a constant profile of $d \Phi_{0} / d \psi$ giving a zero shearing rate $E \times B$ flow (open symbols) and a linear profile of $d \Phi_{0} / d \psi$ giving a nonzero shearing rate $E \times B$ flow (dashed line).

9. Growth rates of helical-ITG modes vs Mach number in a $2 \mathrm{D}$ heliac for a constant profile of $d \Phi_{0} / d \psi$ giving a zero shearing rate $E \times B$ flow (open symbols) and a linear profile of $d \Phi_{0} / d \psi$ giving a nonzero shearing rate $E \times B$ flow (dashed line).

10. Pertubed potential contours for a helical ITG mode without $E \times B$ flow. 
11. Pertubed potential contours for a helical ITG mode with $E \times B$ flow defined with a linear profile of $d \Phi_{0} / d \psi$ with zero value at $s=s_{0}, \operatorname{Mach}=0.01$.

12. Pertubed potential contours for a helical ITG mode with $E \times B$ flow defined with a linear profile of $d \Phi_{0} / d \psi$ with zero value at $s=s_{0}, \mathrm{Mach}=0.05$. 


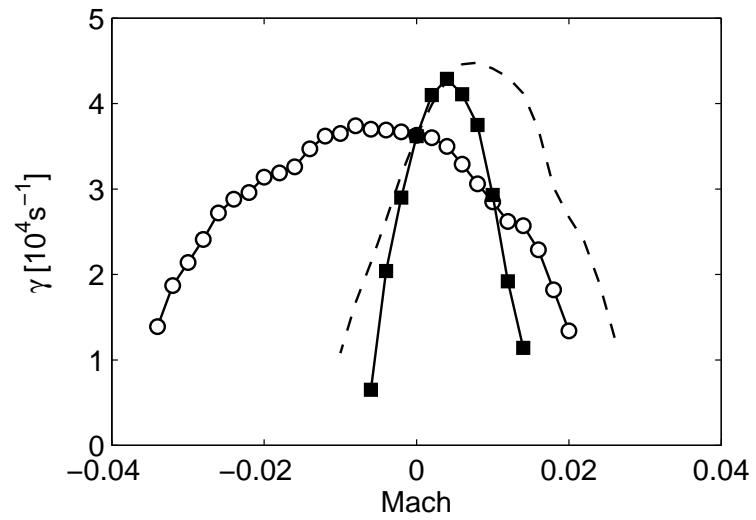

Figure 1: 

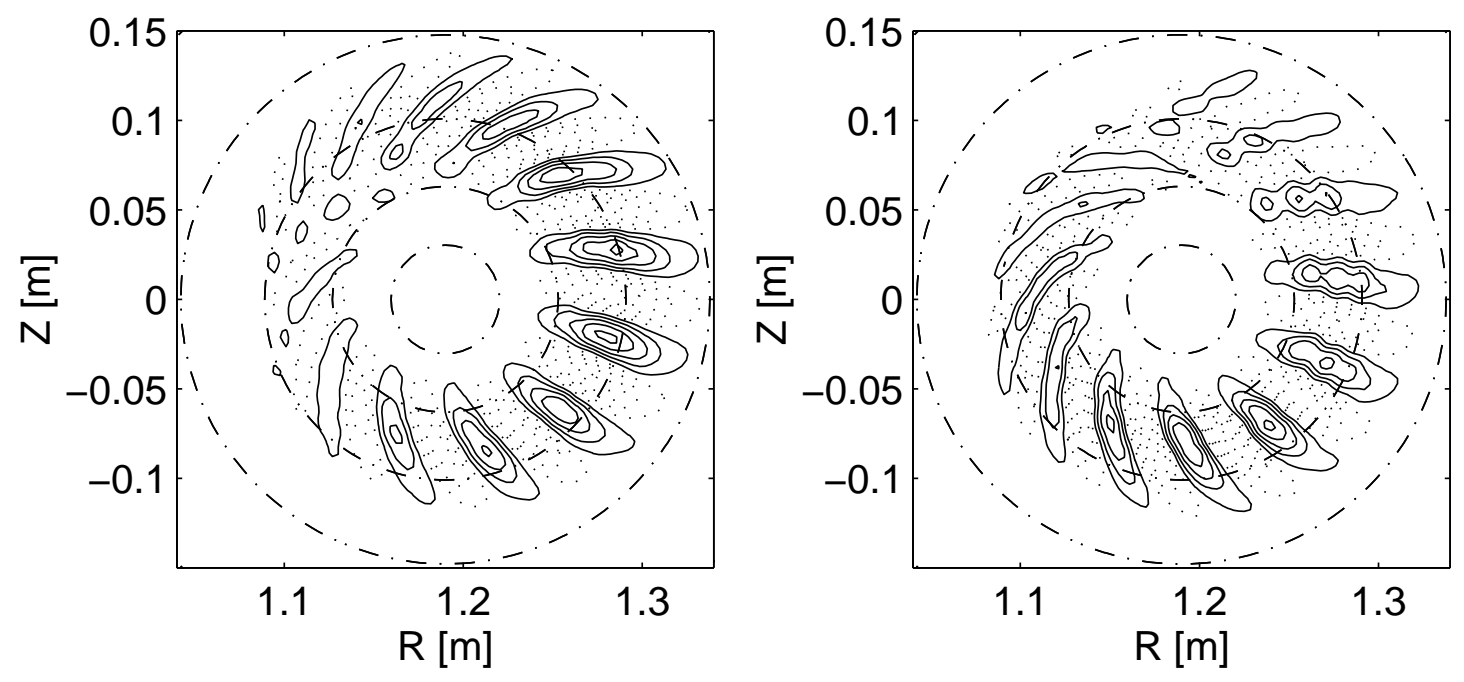

Figure 2: 


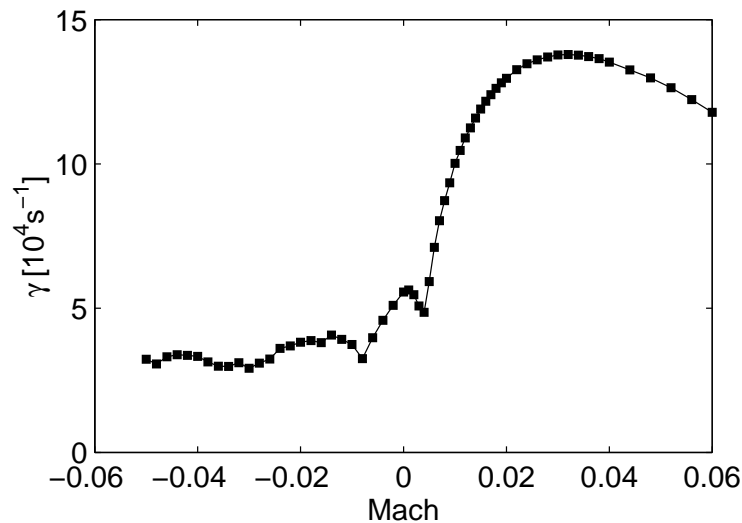

Figure 3: 

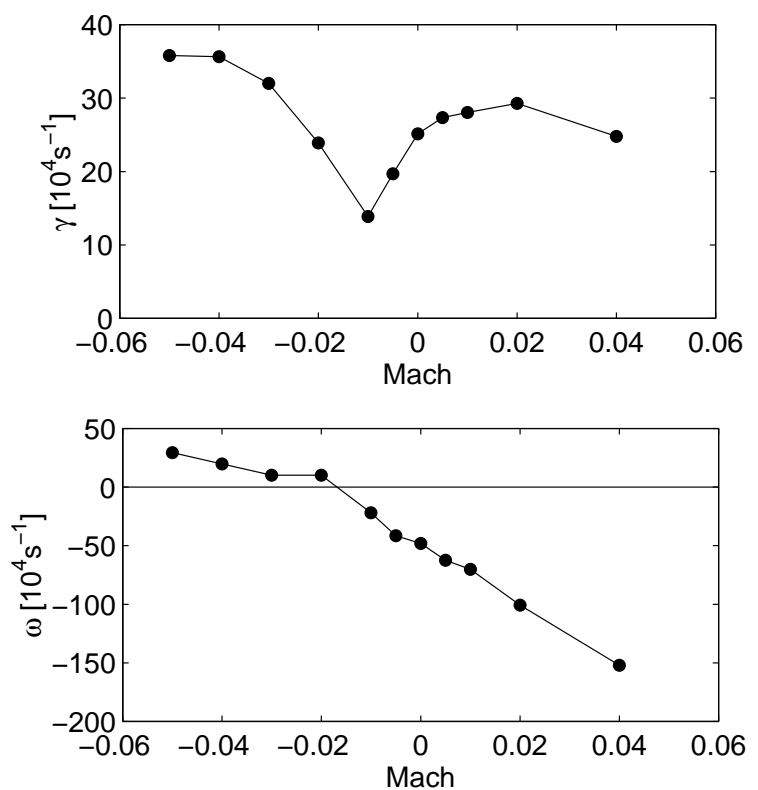

Figure 4: 


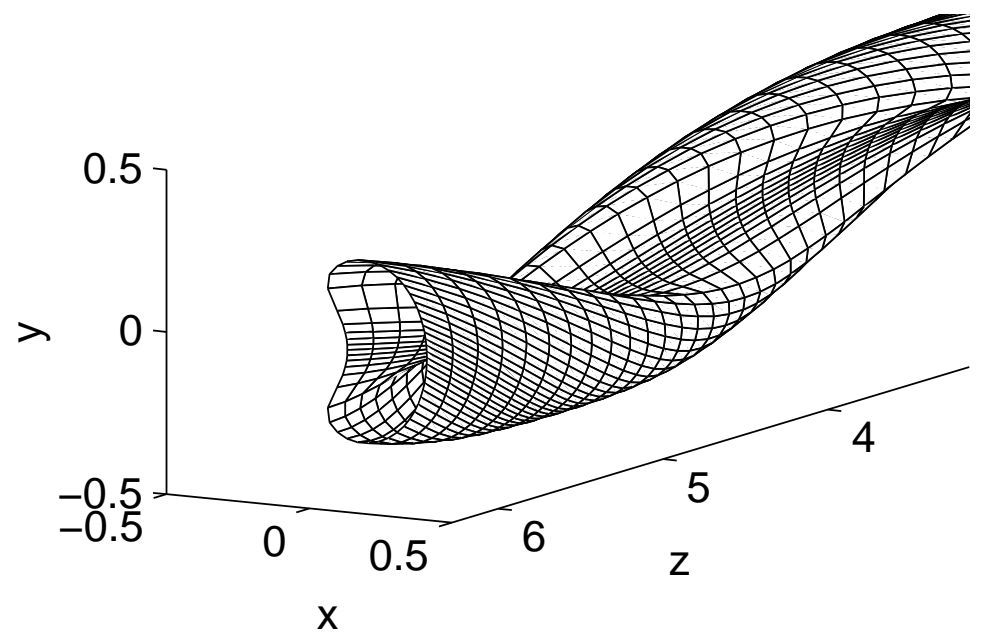

Figure 5: 


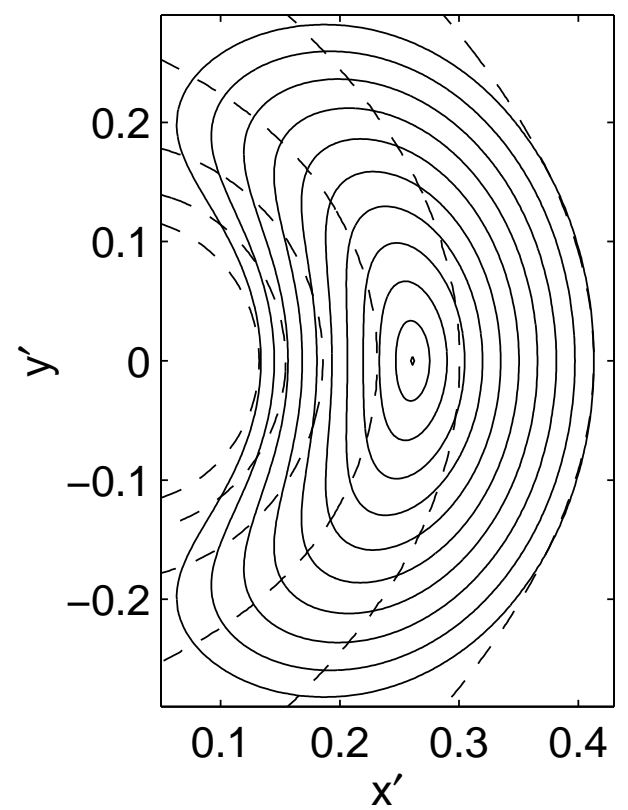

Figure 6: 

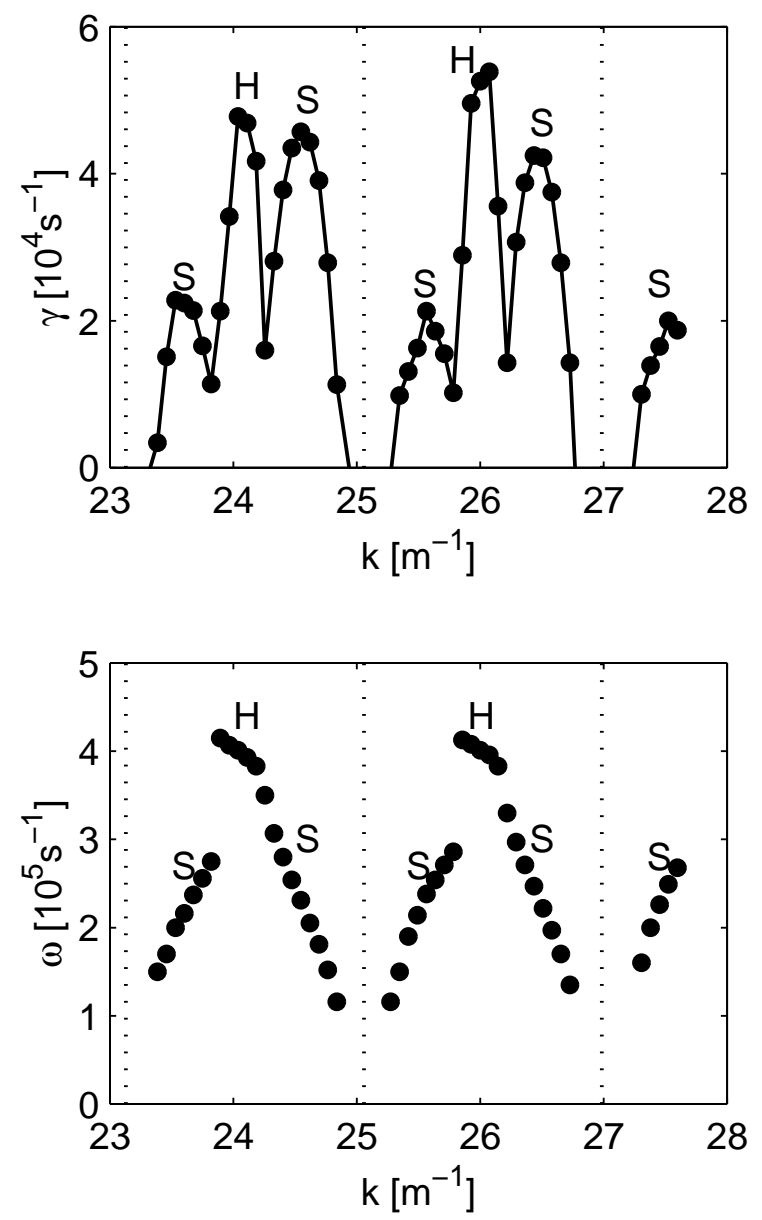

Figure 7: 


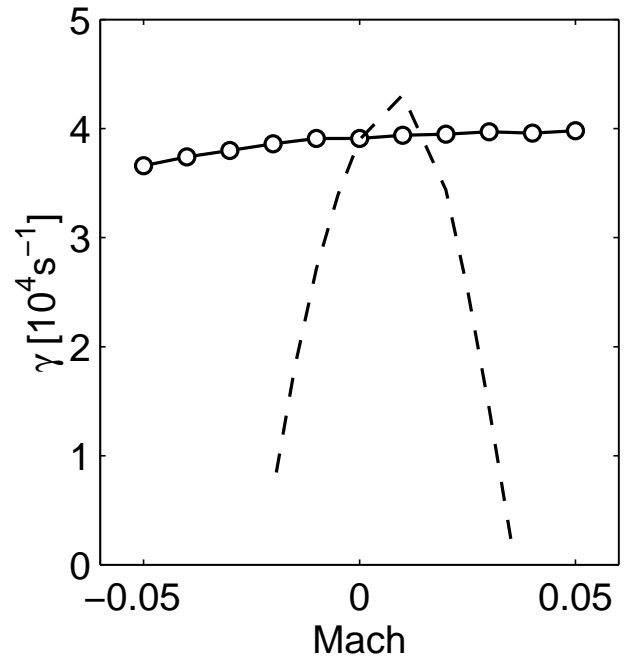

Figure 8: 


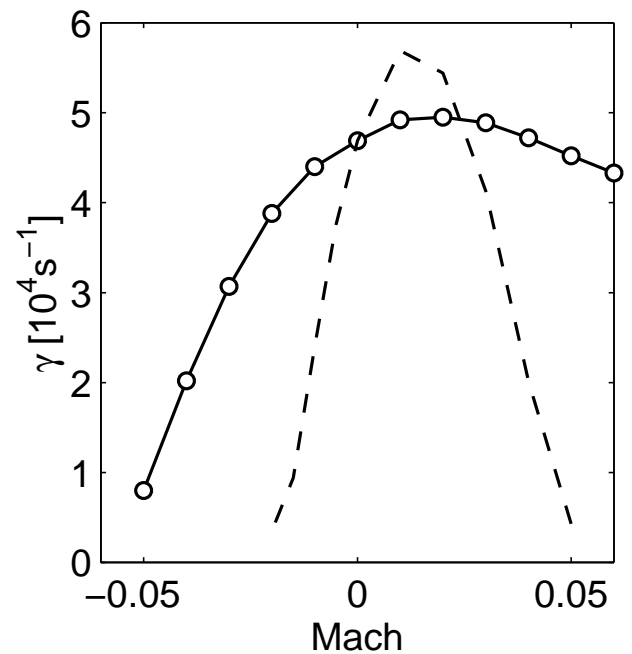

Figure 9: 


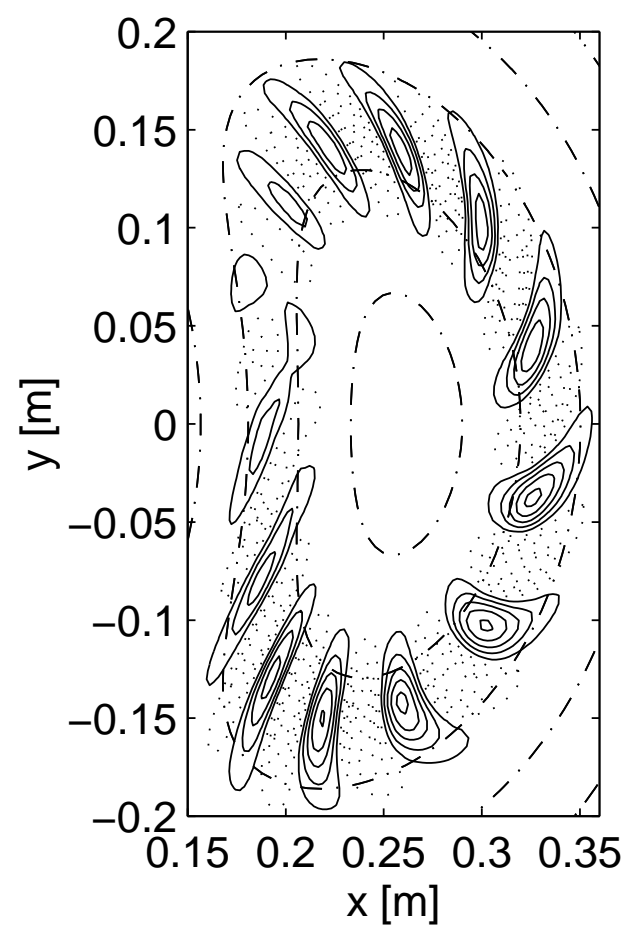

Figure 10: 


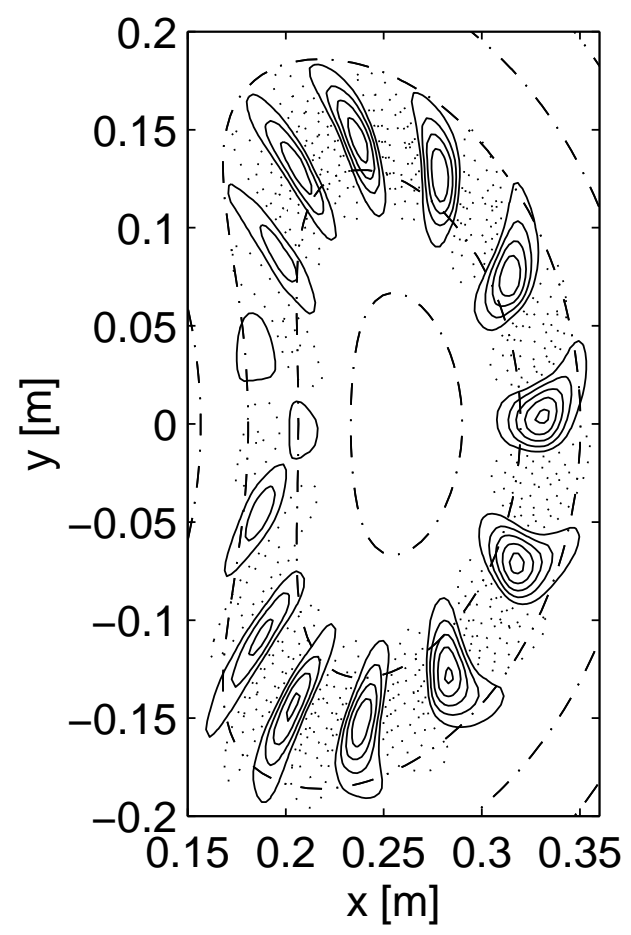

Figure 11: 


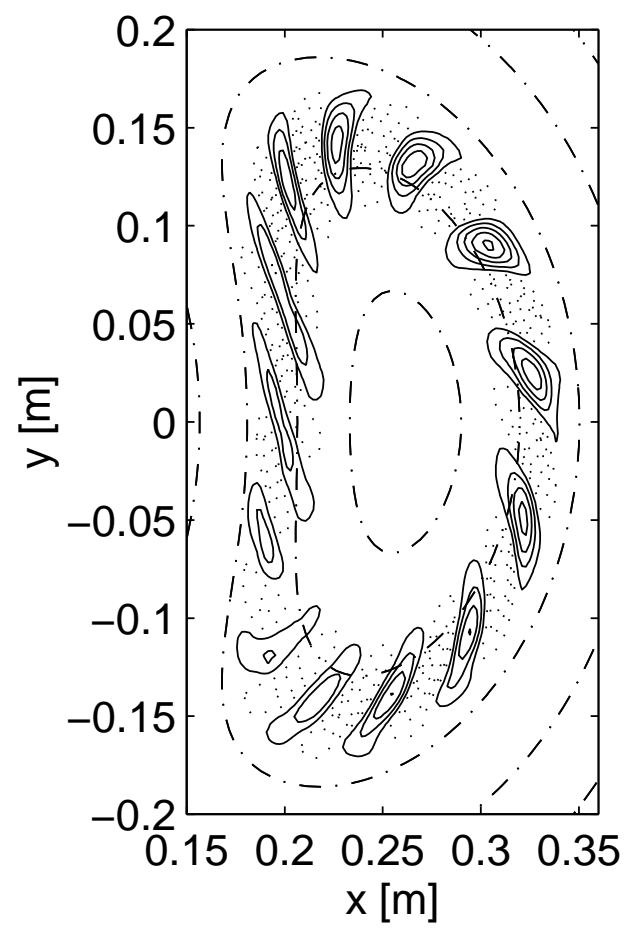

Figure 12: 